\title{
Requirement of NF-кB Activation to Suppress p53-Independent Apoptosis Induced by Oncogenic Ras
}

\author{
Marty W. Mayo, Cun-Yu Wang, Patricia C. Cogswell, \\ Kelley S. Rogers-Graham, Scott W. Lowe, Channing J. Der, \\ Albert S. Baldwin Jr.*
}

The ras proto-oncogene is frequently mutated in human tumors and functions to chronically stimulate signal transduction cascades resulting in the synthesis or activation of specific transcription factors, including Ets, c-Myc, C-Jun, and nuclear factor kappa B (NF-кB). These Ras-responsive transcription factors are required for transformation, but the mechanisms by which these proteins facilitate oncogenesis have not been fully established. Oncogenic Ras was shown to initiate a p53-independent apoptotic response that was suppressed through the activation of NF-кB. These results provide an explanation for the requirement of NF-кB for Ras-mediated oncogenesis and provide evidence that Ras-transformed cells are susceptible to apoptosis even if they do not express the p53 tumor-suppressor gene product. Mutations in a ras allele occur in $30 \%$ of all human tumors (1), making ras the most widely mutated human proto-oncogene. Both mitogen-activated protein (MAP) kinase-dependent and MAP kinase-independent pathways mediate Ras-induced cellular responses (2), and these signal transduction pathways ultimately control the activity of various transcription factors (3). The Ets, c-Myc, and c-Jun proteins are Rasresponsive transcription factors required for cellular transformation in vitro (4) and in vivo (5). The transcription factor NF-кB is

M. W. Mayo and P. C. Cogswell, Lineberger Comprehensive Cancer Center, University of North Carolina School of Medicine, Chapel Hill, NC 27599, USA.

C.-Y. Wang, Lineberger Comprehensive Cancer Center, University of North Carolina School of Medicine; Curriculum in Genetics and Molecular Biology; and Department of Endodontics, University of North Carolina School of Dentistry, Chapel Hill, NC 27599, USA.

K. S. Rogers-Graham, Department of Pharmacology, University of North Carolina School of Medicine, Chapel Hill, NC 27599, USA.

S. W. Lowe, Cold Spring Harbor Laboratory, Cold Spring Harbor, NY 11724, USA.

C. J. Der, Lineberger Comprehensive Cancer Center, University of North Carolina School of Medicine; Curriculum in Genetics and Molecular Biology; and Department of Pharmacology, University of North Carolina School of Medicine, Chapel Hill, NC 27599, USA.

A. S. Baldwin, Lineberger Comprehensive Cancer Center, University of North Carolina School of Medicine; Curriculum in Genetics and Molecular Biology; and Department of Biology, University of North Carolina, Chapel Hill, NC 27599, USA.

*To whom correspondence should be addressed at Lineberger Comprehensive Cancer Center, Campus Box 7295, University of North Carolina, Chapel Hill, NC 27599, USA. also activated in response to oncogenic Ras (6), and this regulation occurs largely through the stimulation of the transcriptional function of the NF- $\kappa$ B RelA/p65 subunit (7). Moreover, NF- $\kappa \mathrm{B}$ is required for Ras-mediated focus-forming activity (7), and activation of this transcription factor provides protection against apoptosis $(8,9)$. Because NF- $\kappa$ B may play a direct role in cellular transformation (10) and because oncogenesis appears to require an anti-apoptotic function (11), we investigated whether oncogenic Ras requires NF- $\mathrm{NB}$ activation to block transformation-induced programmed cell death.

To determine whether the inhibition of NF-кB in Ras-transformed cells would initiate a cell death response, we used $\beta$-galactosidase $(\beta-\mathrm{Gal})$ expression assays to measure cell viability. We inhibited NF-кB activity with a super-repressor form of $\mathrm{I} \kappa \mathrm{B} \alpha$ (SR-I $\mathrm{B} \alpha$ ), which cannot be phosphorylated (12) or degraded (13) and, therefore, blocks the nuclear translocation and subsequent transactivation of $\mathrm{NF}-\kappa \mathrm{B}$-responsive genes (8). Parental NIH 3T3 and H-Rastransformed cells (3T3 H-Ras[V12]) were cotransfected with a pCMV-LacZ reporter and with either an empty expression vector control or a vector encoding SR-ІкB $\alpha$. HRas-transformed NIH 3T3 cells expressing $\mathrm{SR}-\mathrm{I} \kappa \mathrm{B} \alpha$ displayed a decrease in the total

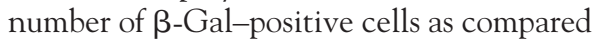
with cells transfected with the vector control (Fig. 1). In contrast, parental NIH 3T3 
cells expressing SR-ІкB $\alpha$ showed no reduction in the number of $\beta$-Gal-positive cells, indicating that the expression from the pCMV-lacZ reporter was not affected by the super-repressor and that expression of $\mathrm{I} \kappa \mathrm{B} \alpha$ did not kill nontransformed parental cells (Fig. 1). Similar results were obtained with H-Ras(V12)-transformed Rat-1 fibroblasts and C127 mouse mammary epithelial cells (14). Because gain-of-function mutations in either $\mathrm{K}$ - or N-ras are more prevalent in human cancers than are mutations in H-ras (1), we examined whether NF-кB served a similar protective role for other oncogenic Ras proteins. Like 3T3 HRas(V12) cells, NIH 3T3 cells transformed with other activated alleles of ras died after the expression of SR-IкB $\alpha$ (Fig. 1). Nontransformed NIH 3T3 cells stably expressing wild-type $\mathrm{H}$-Ras did not exhibit a loss of cell viability in the presence of the SR$\mathrm{I} \kappa \mathrm{B} \alpha(14)$. These results indicate that oncogenic Ras (but not wild-type H-Ras) requires the cell survival function of NF- $\mathrm{B}$ to overcome the activation of a death pathway initiated in transformed cells.

To determine whether the loss of cell viability was a direct effect of oncogenic Ras, we used the Rat-1:iRas cell line, which contains a stably integrated oncogenic Hras(V12) gene under the controlled expression of an isopropyl- $\beta$-D-thiogalactopyrano-

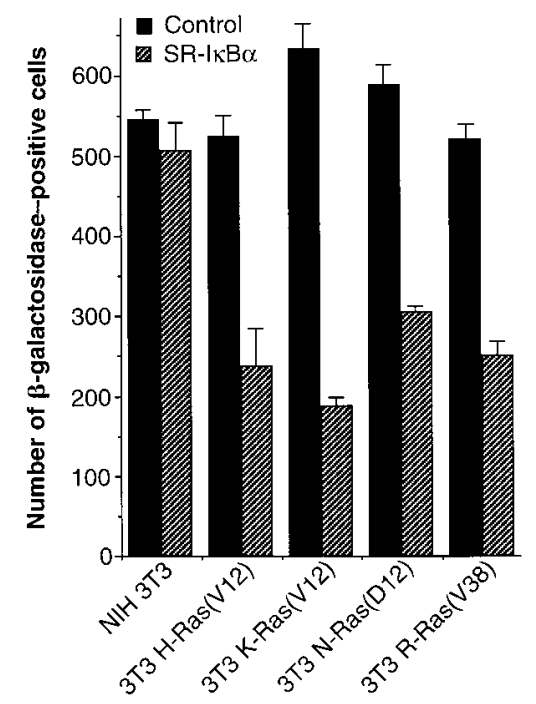

Fig. 1. Effects of SR-IкB $\alpha$ expression in parental and Ras-transformed NIH 3T3 cells. Parental $\mathrm{NIH} 3 \mathrm{~T} 3$ and Ras-transformed NIH 3 T3 cells were cotransfected (8) with pCMV-LacZ (pcDNA3-LacZ, Invitrogen; $0.2 \mu \mathrm{g}$ per 24-well plate) and either an empty expression vector control (CMV-4) or with vector encoding SR$I_{\kappa B} \alpha(1 \mu \mathrm{g}$ per plate each). After 48 hours cells were fixed and stained with X-Gal, and $\beta$-Galpositive cells were counted in each well. The results represent the mean \pm SD of three independent experiments performed in triplicate. side (IPTG)-inducible promoter (15). To inhibit NF-кB activation, we established the Rat-1:iRasI line, which constitutively expresses SR-I $\mathrm{B} \alpha$. Moreover, a control line was established, Rat-1:iRasV, that contains the empty expression vector. The addition of IPTG induced similar amounts of $\mathrm{p} 21^{\text {ras }}$ protein in each of the cell lines, whereas only the Rat-1:iRasI cells displayed a larger immunoreactive band that corresponded to the Flag-tagged SR-IкB $\alpha$ (Fig. $2 \mathrm{~A})$. In contrast with the Rat-1:iRas $\mathrm{V}$ control cells, the Rat-1:iRasI line did not demonstrate increased nuclear NF-кB binding activity or increased transactivation of the $3 \mathrm{x}-\mathrm{\kappa} \mathrm{B}$-dependent reporter in response to
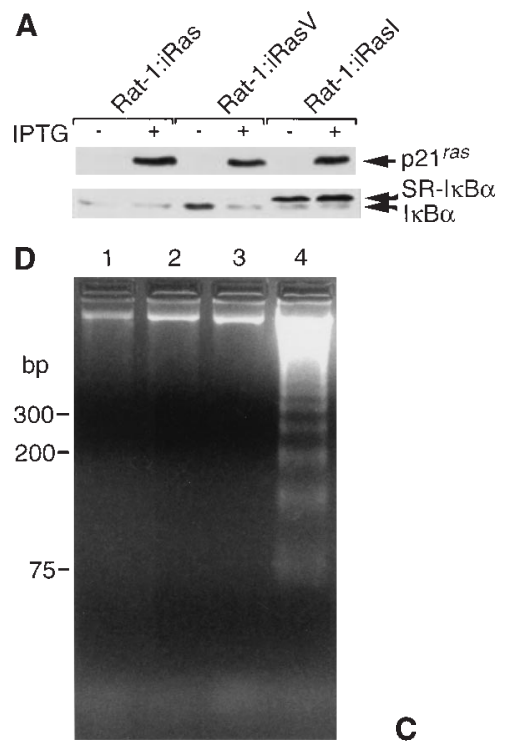

Fig. 2. Apoptotic effects of Ras in Rat-1 cells expressing SR-IкB $\alpha$. (A) Generation and characterization of the Rat-1: iRas cell line expressing SR-

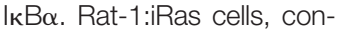
taining an IPTG-inducible $\mathrm{H}$ Ras(V12) cDNA (15), were transfected with the empty expression vector (pCMV-4) or with $\mathrm{pCMV}-4$ containing a cDNA encoding SR-IкB $\alpha$ (1 $\mu \mathrm{g}$ each) and with pBabepuro $(0.2 \mu \mathrm{g})$, which provided puromycin resistance at a concentration of $1 \mu \mathrm{g} / \mathrm{ml}$. The Rat-1:iRasV line contains the empty expression vector, whereas the Rat-1: iRasl line expresses the super-repressor form of $\mathrm{I}_{\kappa} \mathrm{B} \alpha$. Parental Rat-1:iRas and $\mathrm{V}$
C
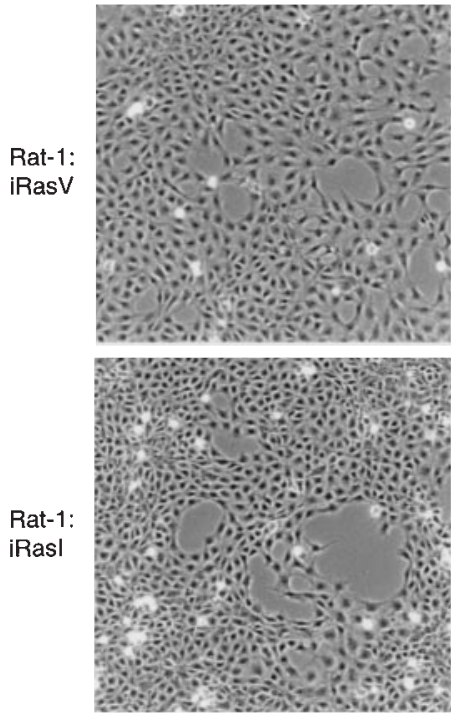

B $\square$ Rat-1:IRasV . Rat-1:IRasV + IPTG $\square$ Rat-1:iRas

Rat-1:iRasI + IPTG

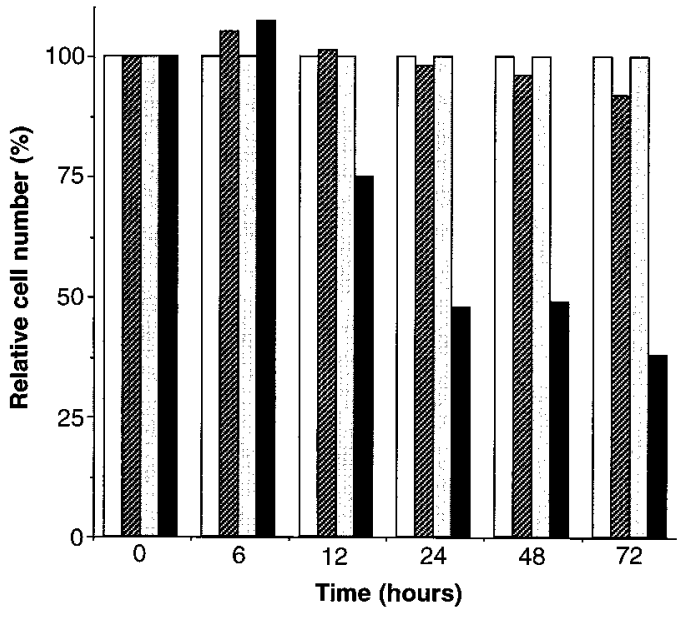

+IPTG

and I cells were treated with 5 mM IPTG (Promega) for 24 hours, and protein expression was assessed by protein immunoblotting total cell extracts (60 $\mu \mathrm{g}$ per lane) with either a pan-Ras antibody (Ab-4, Calbio-

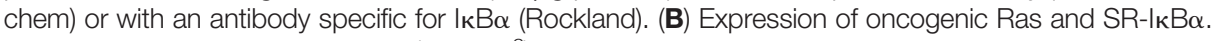
Rat-1:iRasl and Rat-1:iRasV cells $\left(1 \times 10^{6}\right)$ were plated into $100-\mathrm{mm}$ dishes containing Dulbecco's modified Eagle's medium (DMEM) + 10\% fetal bovine serum (FBS), and 12 hours later cells were either left untreated or stimulated with 5 mM IPTG. Cells were harvested at the time indicated, and viable cells were identified by trypan blue dye exclusion. The data represent the mean $(\mathrm{SD} \leq 2.7)$ of three independent experiments. (C) Apoptosis in Rat-1 cells expressing the SR- $\left.\right|_{\kappa} \mathrm{B} \alpha$ and $\mathrm{H}-\mathrm{Ras}(\mathrm{V} 12)$. Micrographs (magnification, $\times 20$ ) of Rat-1:iRasV and Rat-1:iRasl cells grown in DMEM containing $10 \%$ FBS in the absence or presence of IPTG for 36 hours. Dying, nonadherent cells are seen as refractile by phase contrast at this magnification. (D) DNA fragmentation in the absence of NF-кB activation. Rat-1:iRasl and Rat-1:iRasV cells were cultured at $1 \times 10^{5} \mathrm{cells} / \mathrm{ml}$ in DMEM containing 10\% FBS in the absence or presence of IPTG. After 36 hours, cells were harvested, and genomic DNAs were isolated and resolved on 1.5\% agarose gel. Representative DNAs include (lane 1) Rat-1:iRasV, (lane 2) Rat-1:iRasV + IPTG, (lane 3) Rat-1:iRasl, and (lane 4) Rat-1:iRasI + IPTG. Linear double-stranded DNA fragments were sized by means of a 1-kb DNA ladder (Gibco-BRL). 
the expression of oncogenic Ras or tumor necrosis factor stimulation (14).

We used the Rat-1:iRasI cells to determine if the activation of Ras leads to a loss of cell viability under conditions where NF- $\kappa \mathrm{B}$ is inhibited. Rat-1:iRasI and control cells were subjected to IPTG treatment, and total cell numbers were examined over a 3-day period. Greater than a $50 \%$ loss in cell viability was observed in the Rat-1:iRasI cells after Ras expression, as compared with the vector control cells (Fig. 2B). Similar results were obtained when five individual puromycin-resistant clones, expressing similar levels of the IкB $\alpha$ transgene, were pooled and analyzed (14). Both the Rat-1:iRasV and Rat1:iRasI cells displayed distinct Ras-transformed morphology after the addition of IPTG (Fig. 2C) (15). Cell death induced by oncogenic Ras expression in Rat-1:iRasI cells bore the consistent hallmarks of apoptosis (16), including retraction of cellular processes, nuclear condensation, and a loss of adherence to the tissue culture dish (Fig.
2C). Induced expression of activated Ras in Rat-1:iRasI cells, but not the vector control cells, resulted in an increase in genomic DNA fragmentation evident 36 hours after the addition of IPTG, as detected by agarose gel electrophoresis (Fig. 2D). Furthermore, IPTG treatment induced apoptosis, as detected by the appearance of deoxynucleotidyl transferase-mediated dUTP nick-end labeling (TUNEL)-positive cells (14). Fluorescence-activated cell sorter (FACS) analysis of propidium iodide- and bromodeoxyuridine-stained cells confirmed that IPTG did not induce senescence (17) and that the cells that were $<2 \mathrm{~N}$ DNA represented apoptotic cells and not necrotic cells (14). Thus, the induction of oncogenic Ras expression in the presence of a specific inhibitor of NF- $\kappa \mathrm{B}$ leads to enhanced apoptosis. Our results support the hypothesis that oncogenic Ras requires NF-кB activation to block transformation-induced programmed cell death.

The inability of activated Ras to transform primary cells is due, in part, to the

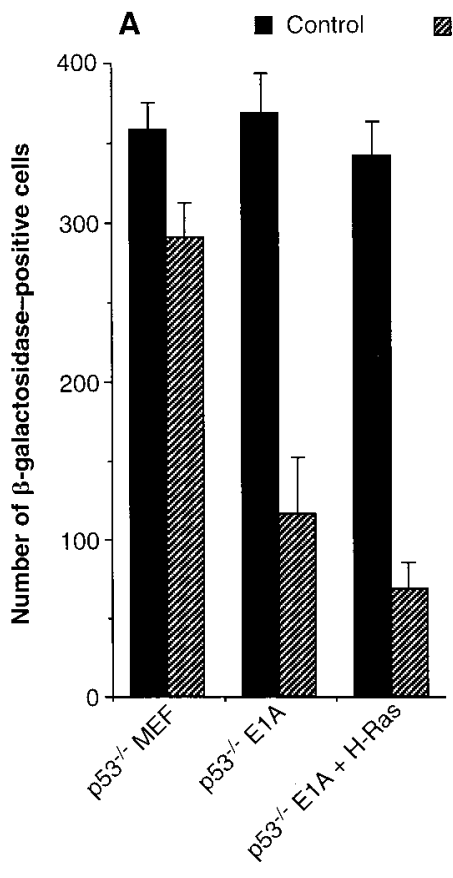

$\mathrm{SR}-\mid \mathrm{KB} \alpha$
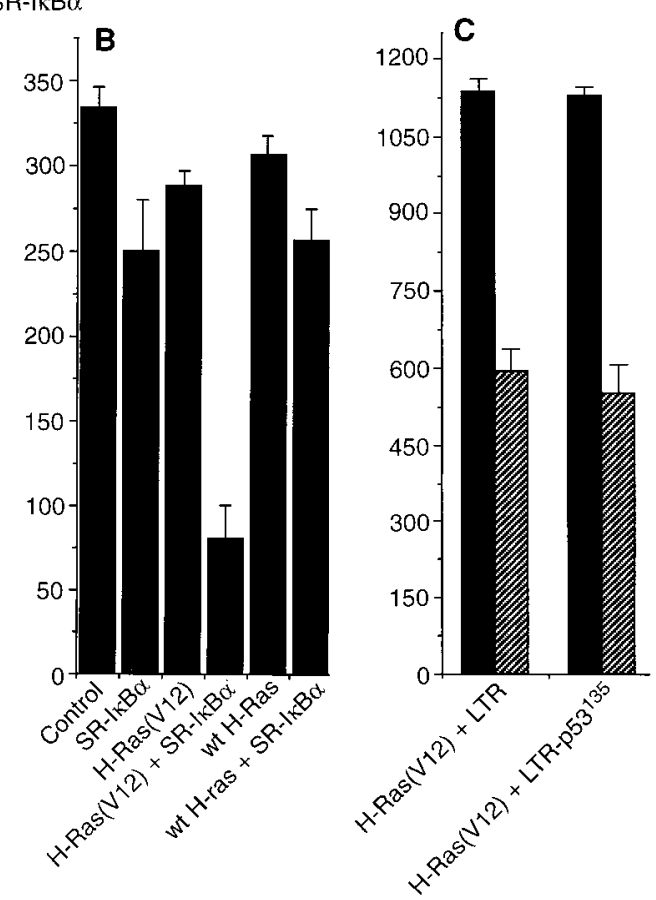

Fig. 3. Ras-mediated apoptosis in cells lacking p53. (A) Requirement of NF-кB in E1A and E1A plus

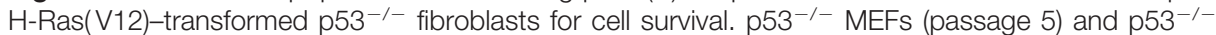
MEFs transformed with either E1A or E1A plus activated $\mathrm{H}$-Ras(V12) were transfected with the pCMVLacZ $(0.2 \mu \mathrm{g})$ and with the empty vector control, or with the SR-IкB $\alpha$ (1 $\mu \mathrm{g}$ each per 24-well plate). After 48 hours cells were fixed and then stained, and $\beta$-Gal-expressing cells were counted. The data are representative of three independent experiments performed in triplicate. (B) Ras-mediated p53-independent cell killing. p53-1- MEFs were transfected with the pCMV-LacZ together with either the empty expression vector control or with the various expression constructs indicated. Cells were stained for $\beta$-Gal activity 48 hours after transfection. Data are shown as the mean \pm SD and are representative of two experiments performed in triplicate. (C) Failure of dominant negative p53 to prevent oncogenic Ras-mediated killing after inactivation of NF-кB. Parental NIH 3T3 cells were transfected with the pCMV-LacZ together with either the vector control (pLTR) or with pLTR-p53 ${ }^{135}$, which encodes the dominant negative p53 mutant. Additionally, cells were transfected with either the empty expression vector control (pCMV-4) or with SR-IкB $\alpha . \beta$-Gal expression assays were performed. Data are shown as the mean \pm SD of two independent experiments performed in triplicate. Bars are shaded as in (A). expression of tumor-suppressor genes, such as

p53 (18) and p16 (19). To determine if p53 had a role in the Ras-dependent killing, we examined whether $\mathrm{p} 53^{-1-}$ mouse embryo fibroblasts (MEFs) expressing oncogenic $\mathrm{H}$ Ras(V12) required the cell survival function of NF-кB. We used p53-/- MEFs expressing either E1A, a viral oncogene product that activates NF-кB (20), or E1A plus H-Ras (21). The cells expressing E1A or E1A and H-Ras displayed enhanced NF-кB-dependent transcriptional activity, as compared with that in control p53-1- MEFs (14). Moreover, the total number of $\mathrm{p} 53^{-1-}$ MEFs expressing E1A or both E1A and H-Ras was reduced after the expression of $\mathrm{SR}-\mathrm{I} \kappa \mathrm{B} \alpha$ (Fig. 3A). In contrast, p53-1- MEFs showed little loss of viability after expression of the super-repressor form of IкB (Fig. 3A). Although E1A induces apoptosis in a p53dependent manner (22), our data indicate that E1A can also induce apoptosis in a $\mathrm{p} 53^{-1-}$-deficient background if the protective function of $\mathrm{NF}-\kappa \mathrm{B}$ is inhibited. To confirm that E1A was not required for Rasmediated killing, we transfected p53-1MEFs with pCMV-LacZ, H-Ras(V12), and with either the empty expression vector or vector encoding $\mathrm{SR}-\mathrm{I} \kappa \mathrm{B} \alpha . \mathrm{p} 53^{-/-} \mathrm{MEFs}$ transfected with oncogenic H-Ras(V12), but not wild-type H-Ras, were effectively killed after inactivation of NF- $\mathrm{B}$ by SR-I $\mathrm{B} \alpha$ (Fig. 3B). Our results indicate that p53 null cells can be effectively transformed by Ras because this oncogene activates $N F-\kappa B$ to suppress apoptosis.

To examine whether both p53-dependent and -independent mechanisms were operative in the Ras-induced apoptosis, we used the mutant $\mathrm{p} 53^{135}$ protein, which functions as a dominant negative inhibitor of endogenously expressed wild-type p53 protein (23). Because p53 is wild-type in NIH 3T3 cells (24), it is possible that p53 contributed to cell death after the expression of oncogenic Ras and SR-IкB $\alpha$ (Fig.1). Parental NIH 3T3 cells were cotransfected with pCMV-LacZ, together with either the vector control (pLTR) or pLTR-p53 ${ }^{135}$, and with either the CMV-4 control vector or the SR-IкB $\alpha$ vector. The dominant negative p $53^{135}$ mutant did not inhibit oncogenic Ras-mediated killing after the inactivation of NF-кB (Fig. 3C). The p53 $3^{135} \mathrm{mu}-$ tant appeared to be functional because it inhibited p53-dependent gene expression in NIH 3T3 cells after doxorubicin treatment (14) and because it blocked p53-dependent apoptosis (25). These results indicate that NF- $\kappa \mathrm{B}$ activity is required to inhibit p53independent programmed cell death mediated by oncogenic Ras.

Our data indicate that oncogenic Ras elicits both pro- and anti-apoptotic pathways, with the latter pathway being domi- 
nant in immortalized rodent cells. Consistent with this idea, Ras has been shown to induce programmed cell death (26) and to suppress apoptosis induced by Myc and E1A $(27,28)$. Our studies demonstrate that the ability of activated Ras to transform p53 null cells is dependent on the ability of this oncogene to activate NF-кB. Thus, there are cell death pathways (independent of p53) that can be initiated by the Ras oncogene after the inactivation of NF-кB. Additional studies indicate that the cell killing induced by Ras is independent of Raf- 1 and its signaling cascade (14). Consistent with this notion, we found that 3T3 R-Ras(V38) cells were killed equally as well as 3T3 H-Ras(V12) cells after expression of the SR-IкB $\alpha$ construct (Fig. 1). Because R-Ras does not interact with the serine-threonine Raf-1 kinase (29), this is consistent with the hypothesis that Raf is not involved in Ras-mediated killing after NF- $\mathrm{B}$ inactivation. Our data demonstrate that oncogenic Ras elicits an apoptotic pathway that is suppressed by the parallel activation of NF$\kappa \mathrm{B}$. Because a complex array of anti-apoptotic proteins are expressed in tumors, the activation of NF- $\mathrm{KB}$ by Ras mutation may play a critical role at the earliest level of tumorigenesis.

\section{REFERENCES AND NOTES}

1. J. L. Bos, Mutat. Res. 195, 255 (1988); F. McCormick, Curr. Opin. Biotechnol. 7, 449 (1996); G. J. Clark and C. J. Der, in GTPases in Biology I, B. F. Dickey and L. Birnbaumer, Eds. (Springer-Verlag, Berlin, 1993), pp. 259-288; I. G. Macara, K. M.

\section{Lounsbury, S. A. Richards, C.
Sagi, FASEB J. 10, 625 (1996).}

2. R. Khosravi-Far et al., Mol. Cell. Biol. 16, 3923 (1996); T. Joneson, M. A. White, M. H. Wigler, D. Bar-Sagi, Science 271, 810 (1996); R.-G. Quin, F. McCormick, M. Symons, Nature 374, 457 (1995); G. C. Prendergast et al., Oncogene 10, 2289 (1995); R.-G. Qiu, J. Chen, F. McCormick, M. Symons, Proc. Natl. Acad. Sci. U.S.A. 92, 11781 (1995); G. J. Clark, J. K. Westwick, C. J. Der, J. Biol. Chem. 272, 1677 (1997).

3. B. Binetruy, T. Smeal, M. Karin, Nature 351, 122 (1991); T. Smeal et al., ibid. 354, 494 (1991); B.-S. Yang et al., Mol. Cell. Biol. 16, 538 (1996); M. Colman and M. Ostrowski, Nucleic Acids Res. 24, 1971 (1996); M. Karin, J. Biol. Chem. 270, 16483 (1995); O. Coso et al., Cell 81, 1137 (1995); A. Minden, A. Lin, F. Claret, A. Abo, M. Karin, ibid., p. 1147.

4. S. Langer, D. Bortner, M. Roussel, C. Sherr, M. Ostrowski, Mol. Cell. Biol. 12, 5355 (1992); M. GrangerSchnarr, E. Benusiglio, M. Schnarr, P. SassoneCorsi, Proc. Natl. Acad. Sci. U.S.A. 89, 4236 (1992); A. Lloyd, N. Yancheva, B. Wasylyk, Nature 352, 635 (1991); M. D. Sklar et al., Mol. Cell. Biol. 11, 3699 (1991).

5. R. Johnson, B. Spiegelman, D. Hanahan, R. Wisdom, Mol. Cell. Biol. 16, 4504 (1996).

6. T. Finco and A. Baldwin, J. Biol. Chem. 268, 17676 (1993).

7. T. S. Finco et al., ibid. 272, 24113 (1997).

8. C.-Y. Wang, M. Mayo, A. Baldwin, Science 274, 784 (1996).

9. A. Beg and D. Baltimore, ibid., p. 782; D. Van Antwerp, S. J. Martin, T. Kafri, D. Green, I. Verma, ibid., p. 787; M. Arsura, M. Wu, G. Sonenshein, Immunity 5, 31 (1996); Z.-G. Liu, H. Hsu, D. Goeddel, M. Karin, Cell 87, 565 (1996)

10. A. Baldwin, Annu. Rev. Immunol. 14, 649 (1996)

11. G. Williams, Cell 65, 1097 (1991); C. Thompson, Science 267, 1456 (1995).

12. J. Brockman et al., Mol. Cell. Biol. 15, 2809 (1995); K. Brown et al., Science 267, 1485 (1995); E. Traenckner et al., EMBO J. 14, 2876 (1995); J. DiDonato et al., Mol. Cell. Biol. 16, 1295 (1996).

13. V. Palombello, O. Rando, A. Goldberg, T. Maniatis, Cell 78, 773 (1994); Z. Chen et al., Genes Dev. 9, 1586 (1995)

14. M. W. Mayo and A. S. Baldwin Jr., unpublished data
M. McMahon, Genes Dev. 9, 1953 (1995).

6. W. Bursch, L. Kleine, M. Tenniswood, Biochem. Cell Biol. 68, 1071 (1990); J. Kerr, C. Winterford, B. Harmon. Cancer 73, 2013 (1994).

17. M. Serrano, A. Lin, M. McCurrach, D. Beach, S. Lowe, Cell 88, 593 (1997).

18. N. Tanaka et al., ibid. 77, 829 (1994).

19. M. Serrano et al., ibid. 85, 27 (1996).

20. M. L. Schmitz et al. , Mol. Cell. Biol. 16, 4052 (1996).

21. S. Lowe, H. Ruley, T. Jacks, D. Housman, Cell 74, 957 (1993).

22. M. Debbas and E. White. Genes Dev. 7, 546 (1993).

23. D. Michalovitz, O. Halevy, M. Oren, Cell 62, 671(1990).

24. M. L. Quo, Y. W. Chou, Y. P. Chau, T. S. Huang, Mol. Carcinog. 18, 221 (1997)

25. M. Holmes-McNary and A. S. Baldwin Jr., unpublished data.

26. C. Chen and D. Faller, Oncogene 11, 1487 (1995); H.-J. Lin, V. Eviner, G. Prendergast, E. White, Mol Cell. Biol. 15, 4536 (1997); H.-G. Wang et al., J. Cell. Biol. 129, 1103 (1995).

27. A. Kauffmann-Zeh et al., Nature 385, 544 (1997)

28. H.-J. Lin, V. Eviner, G. Prendergast, E. White, Mol. Cell. Biol. 15, 4536 (1995).

29. C. Herrmann, G. Horn, M. Spaargaren, A. Witting hofer, J. Biol. Chem. 271, 6794 (1996); S. Huff, L. Quilliam, A. Cox, C. Der, Oncogene 14, 133 (1997); B. Marte, P. Rodriguez-Viciana, S. Wennstrom, P. Warne, J. Downward, Curr. Biol. 7, 63 (1997).

30. We thank Y. Kaziro for permission to use the Rat-1: iRas cells and M. McMahon for providing the cell line; D. Ballard for the super-repressor $\left.\right|_{\kappa} B \alpha$ construct; $M$. Ostrowski for the Ets-transformed cell line; S. Drouin for immunoblotting; L. Arnold and J. Watson for FACS analysis; E. Reap for helpful discussions; and S. Earp and D. Guttridge for reading the manuscript and for helpful suggestions. Supported by $\mathrm{NIH}$ grant CA72771 and by the Department of the Army grant DAMD17-94-J-4053 to A.S.B. C.J.D. and S.W.L. are supported by $\mathrm{NIH}$ grants CA52072 and CA13106, respectively. M.W.M. is supported by NIH postdoctoral fellowship 1F32-CA69790. C.-Y.W. is supported by a Dentist Scientist Award from NIH.

20 March 1997; accepted 30 October 1997

\section{SCIIENCE}

Note to

Readers

about

Technical

Comments

\section{Beginning in 1998, summaries of technical comments will appear in the printed journal; full text and figures will appear only online.}

\section{This change will allow:}

Free access to the full text of technical comments and responses for all Science Online readers.

n Quicker publication of reviewed comments, criticisms, and responses.

- Linkage between reports and comments, which would notify readers that subsequent comments were published about a report (or article) in Science.

- More comprehensive figures and tables in the comments and more detailed discussion of methods and instrumentation.

- Up-front coverage in summaries of the comments and responses, which will appear in "This Week in Science" in the printed journal, with Web addresses for the full texts.

- Continued full citation, with technical comment titles and authors continuing to be listed in the printed Table of Contents, and authors continuing to be cited in the quarterly online index. 


\section{Science}

\section{Requirement of NF-kB Activation to Suppress p53-Independent Apoptosis Induced by Oncogenic}

Ras

Marty W. Mayo, Cun-Yu Wang, Patricia C. Cogswell, Kelley S. Rogers-Graham, Scott W. Lowe, Channing J. Der and Albert S. Baldwin Jr.

Science 278 (5344), 1812-1815.

DOI: $10.1126 /$ science.278.5344.1812

ARTICLE TOOLS http://science.sciencemag.org/content/278/5344/1812

REFERENCES This article cites 48 articles, 22 of which you can access for free http://science.sciencemag.org/content/278/5344/1812\#BIBL

PERMISSIONS $\quad$ http://www.sciencemag.org/help/reprints-and-permissions 